\title{
HULC and H19 Played Different Roles in Overall and Disease-Free Survival from Hepatocellular Carcinoma after Curative Hepatectomy: A Preliminary Analysis from Gene Expression Omnibus
}

\author{
Zongguo Yang, ${ }^{1}$ Yunfei Lu, ${ }^{1}$ Qingnian Xu, ${ }^{1}$ Bozong Tang, \\ Cheol-Keun Park, ${ }^{2}$ and Xiaorong Chen ${ }^{1}$ \\ ${ }^{1}$ Shanghai Public Health Clinical Center, Fudan University, Shanghai 201508, China \\ ${ }^{2}$ Samsung Medical Center, Sungkyunkwan University School of Medicine, Seoul 135-710, Republic of Korea \\ Correspondence should be addressed to Cheol-Keun Park; ckpark@skku.edu and Xiaorong Chen; xiaorong3chen@163.com
}

Received 12 March 2015; Accepted 28 May 2015

Academic Editor: Marco E. M. Peluso

Copyright (C) 2015 Zongguo Yang et al. This is an open access article distributed under the Creative Commons Attribution License, which permits unrestricted use, distribution, and reproduction in any medium, provided the original work is properly cited.

\begin{abstract}
Objective. This study aimed to evaluate the relationships between long noncoding RNAs (lncRNAs) in tumor tissues and hepatocellular carcinoma (HCC) aggressiveness and survival. Methods. We correlated the lncRNAs in tumor tissues with HCC survival and clinicopathological features based on Gene Expression Omnibus expression profile GSE36376. Results. Eight lncRNAs and $240 \mathrm{HCC}$ patients were included. Cox regression analysis indicated that HULC was a positive factor for HCC overall survival $(\mathrm{HR}=0.885$, $95 \% \mathrm{CI}=0.797-0.983$, and $P=0.023)$ and disease-free survival time $(\mathrm{HR}=0.913,95 \% \mathrm{CI}=0.835-0.998$, and $P=0.045)$. $\mathrm{H19}$ and UCA1 were both demonstrated to be risk factors of HCC disease-free survival in multivariate Cox model $(\mathrm{HR}=1.071,95 \% \mathrm{CI}=1.01-$ 1.137, and $P=0.022$ and $\mathrm{HR}=2.4,95 \% \mathrm{CI}=1.092-5.273$, and $P=0.029$, resp.). But Kaplan-Meier method showed no significant association between UCA1 and HCC disease-free survival (log rank $P=0.616$ ). Logistic regression demonstrated that H19 was overexpressed in HBV-infected patients $(\mathrm{OR}=1.14,95 \% \mathrm{CI}=1.008-1.29$, and $P=0.037)$. HULC had a significant association with vascular invasion $(\mathrm{OR}=0.648,95 \% \mathrm{CI}=0.523-0.803$, and $P<0.001) . \mathrm{H} 19$ and MEG3 were both considered to be risk factors for high AFP level $(\mathrm{OR}=1.45,95 \% \mathrm{CI}=1.277-1.646$, and $P<0.001$ and $\mathrm{OR}=1.613,95 \% \mathrm{CI}=1.1-2.365$, and $P=0.014$, resp. $)$. Conclusions. Contributing to decreased susceptibility to vascular invasion, upregulation of HULC in tumor tissues was positively associated with HCC survival. In contrast, H19 overexpression might be risk factor for HCC aggressiveness and poor outcomes.
\end{abstract}

\section{Introduction}

Globally, HCC is the most common primary liver cancer, the fifth most common cancer, and the third most common cause of cancer-related deaths, representing around $5 \%$ of all cancers [1]. The occurrence and metastasis of HCC is a multistep process including tumor clinicopathological features, changes in signal transduction pathways, environmental makeup, gene mutations, and gene regulations [2, 3]. Recently, the widespread search for effective biomarkers of HCC is hoped to lead to earlier diagnosis and improve prognosis by allowing earlier intervention [3]. However, the molecular mechanism of HCC, especially gene regulatory mechanism, has not yet been fully elucidated.
It is well known that more than $90 \%$ of human genome undergoes transcription but does not code for proteins. lncRNAs are a class of noncoding RNA transcripts longer than 200 nucleotides with little or no protein-coding capacity [4]. As one of the key members of gene regulatory networks, lncRNAs take part in epigenetic regulation and are involved in diverse biological processes as well as disease pathogenesis $[5,6]$. It is proved that lncRNAs can be used as potential carcinogenic and anticarcinogenic RNA [7]. In recent years, several reports revealed that lncRNAs were dysregulated in cancers [8-10], though their specific role in cancer proliferation, development, and progression was largely unclear. Increasing evidence has indicated that IncRNAs play a critical 
role in tumor biology, including tumor initiation, progression, and metastasis [11-13]. In addition, recent studies indicated that a number of lncRNAs are dysregulated in HCC, while their aberrant expressions are associated with tumorigenesis, metastasis, and diagnosis. Unfortunately, few data focused on the association of lncRNAs with prognosis of HCC $[14,15]$. On the other hand, the roles of lncRNAs in HCC aggressiveness were controversial [10, 14].

Considering that increased evidence relates changes in expression levels of lncRNAs to cancers and controversial conflict existed before and few studies have evaluated the role of lncRNAs in HCC patients, further analysis to clarify this relationship between lncRNAs and HCC prognosis is urgently needed. This study set out to define the relationships between lncRNAs and survival and clinicopathological features in HCC patients, in the hope that the data may provide novel biomarker candidates as well as useful insights into the pathogenesis and progression of HCC.

\section{Methods}

2.1. Patients. 240 tumor tissues containing no necrosis or hemorrhage were available from primary HCC patients who were treated with surgical resection or liver transplantation at Samsung Medical Center, Seoul, Korea, from July 2000 to May 2006. None of the patients received preoperative chemotherapy. Informed consent was obtained from each patient included in the study, and this study was approved by the institutional review board of Samsung Medical Center, Seoul, Korea, which is consistent with reports by Lim et al. [16]. The second analysis protocol was approved by the Ethics Committee of Shanghai Public Health Clinical Center, Fudan University.

2.2. Source of Data. Tumor tissues of HCC patients after curative hepatectomy in this study were profiled using Illumina HumanHT-12 V4.0 expression beadchip (Illumina Inc., San Diego, CA). The expression data was retrieved from Gene Expression Omnibus (GSE36376, http://www.ncbi.nlm.nih .gov/geo/) [16]. We restricted our search to genes within the lncRNAs, and eight lncRNAs previously reported in chronic liver diseases were included in our analysis.

2.3. End Points. The overall survival was defined as time from surgery to the date of death or last follow-up. The disease-free survival was defined as time from surgery to the date of tumor recurrence or death. The censoring time was defined as the final documented date of no evidence of tumor recurrence by imaging. As presented by Lim et al. [16], clinicopathological features of HCC patients including vascular invasion, major portal vein invasion, intrahepatic metastasis, multicentric occurrence, and nontumor liver pathology were all considered. Patient serum $\alpha$-fetoprotein levels were evaluated and three phase dynamic computed tomography scans were performed at least once every 3 months after surgery until December 31, 2010. When tumor recurrence was suspected, precise diagnostic imaging was performed by magnetic resonance imaging.
TABLE 1: Baseline characteristics of HCC patients.

\begin{tabular}{lc}
\hline Characteristics & $n=240$ \\
\hline Male, $n$ (\%) & $199(82.9)$ \\
Age, median (range), years & $53(45-61)$ \\
Body mass index, mean \pm SD, kg/m ${ }^{2}$ & $24.2 \pm 2.8$ \\
Etiology, HBV/HCV/alcohol/NA, $n$ & $186 / 20 / 14 / 20$ \\
Tumor size, median (range), cm & $3.7(2.5-6.15)$ \\
Vascular invasion, $n$ (\%) & $133(55.4)$ \\
Major portal vein invasion, $n(\%)$ & $9(3.8)$ \\
Intrahepatic metastasis, $n$ (\%) & $55(22.9)$ \\
Multicentric occurrence, $n(\%)$ & $13(5.4)$ \\
Direct invasion of adjacent organ, $n(\%)$ & $5(2.1)$ \\
AJCC stage, I/II/III/IV, $n$ & $102 / 100 / 33 / 5$ \\
BCLC stage, A/B/C, $n$ & $139 / 91 / 10$ \\
$\alpha$-Fetoprotein $>200$ ng/mL, $n(\%)$ & $87(36.3)$ \\
Liver histology of nontumor tissues, $n(\%)$ & \\
$\quad$ Cirrhosis & $115(47.9)$ \\
Chronic active hepatitis & $58(24.2)$ \\
Chronic persistent hepatitis & $36(15.0)$ \\
Reactive hepatitis & $11(4.6)$ \\
Alcoholic hepatitis & $11(4.6)$ \\
\hline
\end{tabular}

NA: not available; HBV: hepatitis B virus; $\mathrm{HCV}$ : hepatitis $\mathrm{C}$ virus; $\mathrm{AJCC}$ : American Joint Committee on Cancer; BCLC: Barcelona Clinic Liver Cancer.

2.4. Statistical Analysis. Student's $t$-test was used to compare means for normally distributed continuous data; MannWhitney $U$ test was used for nonnormally distributed continuous data. Factors associated with the outcomes and clinicopathological features were assessed by univariate analysis and multivariate analysis separately using Cox and logistic regression. Only covariates significantly associated with outcomes at univariate analysis (two-sided $P<0.10$ ) were included in the multivariate model. Results were reported as hazard ratios (HR) or odd ratios (OR) with $95 \%$ confidence intervals (CI). The Kaplan-Meier method was used to compare overall survival between different groups, and the log rank test was used to estimate the difference in survival. ROC curve was performed to evaluate predictive values of potential factors for HCC survival. Statistical analyses were performed using PASW Statistics software version 18.0 from SPSS Inc. (Chicago, IL, USA). All statistical tests were two-tailed, and differences with $P<0.05$ were considered statistically significant.

\section{Results}

3.1. Patient Characteristics. As shown in Table 1 , of the 240 patients included, $82.9 \%(199 / 240)$ were men and $17.1 \%$ (41/240) women with a median age of 53 (45-61) years and a mean body mass index (BMI) $24.2 \pm 2.8 \mathrm{~kg} / \mathrm{m}^{2}$. $77.5 \%(186 / 240)$ of patients had evidence of hepatitis B virus (HBV) infection, 8.3\% (20/240) had evidence of hepatitis C virus (HCV) infection, and 5.8\% (14/240) had evidence of alcohol use and for 20 patients no information was available. 
TABLE 2: LncRNA expression between tumor tissues and nontumor tissues of HCC patients.

\begin{tabular}{lccc}
\hline LncRNAs & Tumor tissues & Nontumor tissues & $P$ value \\
\hline H19, median (range) & $9.28(6.10-14.54)$ & $10.65(7.58-13.83)$ & $<0.001$ \\
HOTAIR, mean \pm SD & $6.19 \pm 0.15$ & $6.20 \pm 0.14$ & 0.448 \\
MEG3, median (range) & $5.98(5.56-11.15)$ & $5.92(5.64-9.30)$ & $\mathbf{0 . 0 0 1}$ \\
MALAT1, mean \pm SD & $5.82 \pm 0.13$ & $5.84 \pm 0.14$ & 0.138 \\
HULC, median (range) & $11.87(6.06-14.23)$ & $11.77(7.80-12.80)$ & 0.085 \\
UCA1, median (range) & $6.51(6.14-7.62)$ & $6.51(6.06-6.90)$ & 0.373 \\
HOXA13, median (range) & $6.62(6.11-8.57)$ & $6.45(5.92-6.81)$ & $<.001$ \\
KCNQ1OT1, median (range) & $6.23(5.45-10.21)$ & $6.17(5.63-6.63)$ & $\mathbf{0 . 0 0 4}$ \\
\hline
\end{tabular}

TABLE 3: Univariate and multivariate Cox regression analysis of lncRNAs associated with overall survival of HCC patients.

\begin{tabular}{lccc}
\hline LncRNAs & Univariate analysis, HR $(95 \%$ CI $)$ & $P$ value & Multivariate analysis, HR (95\% CI) \\
\hline H19, per increase of 1 unit & $1.025(0.955-1.101)$ & 0.488 & \\
HOTAIR, per increase of 1 unit & $3.059(0.788-11.873)$ & 0.106 & \\
MEG3, per increase of 1 unit & $1.079(0.884-1.318)$ & 0.455 & \\
MALAT1, per increase of 1 unit & $3.009(0.673-13.45)$ & 0.149 & $0.085(0.797-0.983)$ \\
HULC, per increase of 1 unit & $0.859(0.773-0.955)$ & 0.117 & \\
UCA1, per increase of 1 unit & $1.986(0.842-4.685)$ & 0.159 & \\
HOXA13, per increase of 1 unit & $0.715(0.449-1.14)$ & 0.35 & \\
KCNQ1OT1, per increase of 1 unit & $0.753(0.415-1.366)$ & \\
\hline
\end{tabular}

HR: hazard ratios; CI: confidence intervals.

The median tumor size was $3.7(2.5-6.15) \mathrm{cm} .55 .4 \%(133 / 240)$ of patients had evidence of vascular invasion, $3.8 \%(9 / 240)$ had evidence of major portal vein invasion, $22.9 \%(55 / 240)$ had evidence of intrahepatic metastasis, and 5.4\% (13/240) had evidence of multicentric occurrence. $2.1 \%(5 / 240)$ had direct invasion of adjacent organ. 36.3\% (87/240) of these patients had an alpha-fetoprotein level more than $200 \mathrm{ng} / \mathrm{mL}$ and $47.9 \%$ (115/240) had a history of cirrhosis, $24.2 \%(58 / 240)$ had chronic active hepatitis, and 15\% (36/240) had chronic persistent hepatitis. Tumor staging including AJCC and BCLC is also described in Table 1.

3.2. LncRNAs Expression Levels. Eight lncRNAs including H19, HOTAIR, MEG3, MALAT1, HULC, UCA1, HOXA13, and KCNQ1OT1 were considered in this analysis. LncRNAs expression levels between tumor and nontumor tissue of HCC patients are shown in Table 2. HOTAIR, MALAT1, HULC, and UCA1 were similarly expressed between tumor tissues and nontumor tissues in HCC patients $(P=$ $0.448,0.138,0.085$, and 0.373 , resp.). MEG3, HOXA13, and KCNQ1OT1 were all overexpressed in HCC tumor tissues $(P=0.001, P<0.001$, and $P=0.004$, resp. $)$. However, H19 was expressed relatively higher in nontumor tissues compared to those in tumor tissues of HCC patients $(P<0.001)$.

3.3. HULC Was Associated with HCC Overall Survival. All lncRNAs included in the analysis were summarized in Table 3. Univariate analysis showed that HULC was a factor associated with overall survival in HCC patients $(P=0.005)$. When all these lncRNAs were evaluated by a multivariate model using enter selection, HULC was indicated to be a positive factor for HCC overall survival $(\mathrm{HR}=0.885,95 \%$ $\mathrm{CI}=0.797-0.983$, and $P=0.023$ ).

We performed a Kaplan-Meier event analysis grouping by HULC identified to be significantly associated with survival presented above. For HULC, we grouped by median expression into a low expression and a high expression group with an 11.87 cut-off. As shown in Figure 1, this revealed that the higher the HULC expression, the greater the chance for longer survival (mean survival time, high $=96.82 \pm 3.70$ and low $=71.52 \pm 4.49$ months, resp.; $\log \operatorname{rank} P<0.001$, Figure 1(A)). ROC curve also demonstrated that HULC expression level in tumor tissues could significantly predict $\mathrm{HCC}$ overall survival (area under $\mathrm{ROC}=0.608 ; P=0.004$, Figure 1(a)).

3.4. H19, HULC, and UCA1 Were Associated with HCC Disease-Free Survival. Table 4 summarizes results from univariate and multivariate regression analyses of potential lncRNAs associated with HCC disease-free survival. H19, HULC, UCA1, and HOXA13 were all factors associated with diseasefree survival in HCC patients (all $P<0.10$ ). Furthermore, multivariate analysis using forward selection has shown that HULC should play a positive role in prolonging HCC diseasefree survival time $(\mathrm{HR}=0.913,95 \% \mathrm{CI}=0.835-0.998$, and $P=0.045)$. However, $\mathrm{H} 19$ and UCA1 were both demonstrated to be risk factors for HCC disease-free survival in multivariate Cox model $(\mathrm{HR}=1.071,95 \% \mathrm{CI}=1.01-1.137$, and $P=0.022$ and $\mathrm{HR}=2.4,95 \% \mathrm{CI}=1.092-5.273$, and $P=0.029$; resp.).

A Kaplan-Meier event analysis using log rank method was also performed further. For H19, HULC, and UCA1, we grouped by median expression into a low expression and a high expression group with cut-offs of 9.28, 11.87, and 6.51, 
TABLE 4: Univariate and multivariate Cox regression analysis of lncRNAs associated with disease-free survival of HCC patients.

\begin{tabular}{lccc}
\hline LncRNAs & Univariate analysis, HR $(95 \%$ CI $)$ & $P$ value & Multivariate analysis, HR (95\% CI) \\
\hline H19, per increase of 1 unit & $1.068(1.007-1.133)$ & 0.028 & $1.071(1.01-1.137)$ \\
HOTAIR, per increase of 1 unit & $2.206(0.711-6.842)$ & 0.171 & 0.022 \\
MEG3, per increase of 1 unit & $1.113(0.947-1.307)$ & 0.193 & \\
MALAT1, per increase of 1 unit & $2.765(0.785-9.748)$ & 0.027 & $0.913(0.835-0.998)$ \\
HULC, per increase of 1 unit & $0.903(0.825-0.989)$ & 0.033 & $2.4(1.092-5.273)$ \\
UCA1, per increase of 1 unit & $2.352(1.071-5.167)$ & 0.06 & $\mathbf{0 . 0 4 5}$ \\
HOXA13, per increase of 1 unit & $0.697(0.478-1.016)$ & 0.632 & $\mathbf{0 . 0 2 9}$ \\
KCNQ1OT1, per increase of 1 unit & $1.116(0.712-1.75)$ & \\
\hline
\end{tabular}

HR: hazard ratios; CI: confidence intervals.

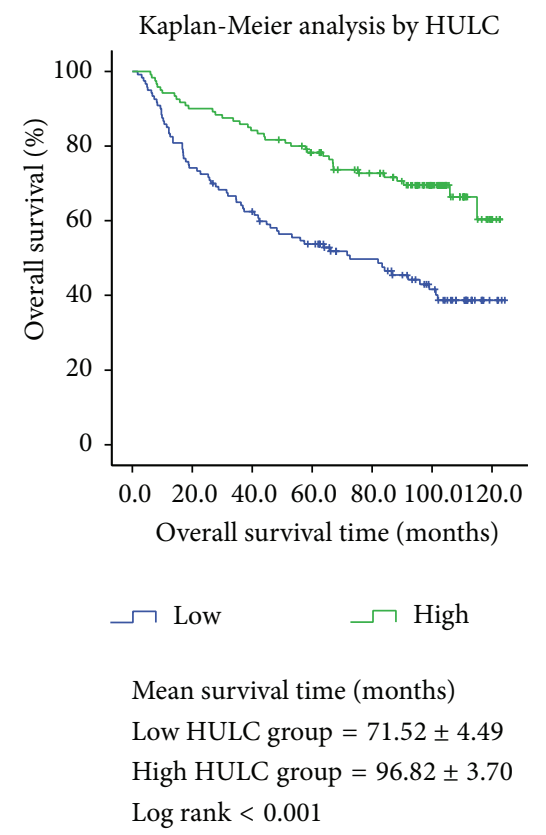

(A)

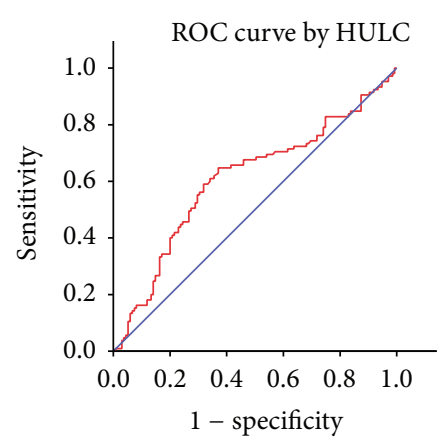

Area under $\mathrm{ROC}=0.608$

$P=0.004$

(a)

FIGURE 1: (A) Kaplan-Meier analysis of HCC overall survival by HULC with median cut-off; (a) ROC curve of HULC for predicting HCC overall survival. respectively. As shown in Figure 2, this indicated that H19 overexpression in HCC tumor tissues might be a risk factor associated with HCC disease-free survival (mean survival time, high $=41.76 \pm 4.35$ and low $=62.54 \pm 4.87$ months, resp.; log rank $P=0.002$, Figure $2(\mathrm{~A})$ ). In contrast, high HULC expression level is contributed to a better diseasefree survival in HCC patients (mean survival time, high = $62.37 \pm 4.73$ and low $=40.98 \pm 4.46$ months, resp.; log rank $P<0.001$, Figure 2(B)). Despite a significant correlation between UCA1 expression and HCC disease-free survival both in univariate and in multivariate regression analyses, the extent of the association by Kaplan-Meier event analysis with log rank method showed no significance (mean survival time, high $=50.17 \pm 4.71$ and low $=52.92 \pm 4.64$ months, resp.; log rank $P=0.616$, Figure $2(\mathrm{C})$ ). ROC curves revealed that $\mathrm{H} 19$ and HULC levels well predict HCC disease-free survival (area under ROC $=0.608, P=0.005$, and area under $\mathrm{ROC}=0.578$, $P=0.042$, resp., Figures 2(a) and 2(b)), while no significance was found between UCA1 and HCC disease-free survival by ROC curve (area under ROC $=0.541, P=0.282$, Figure 2(c)).

3.5. Relationship between LncRNAs and HCC Clinicopathological Features. Only $\operatorname{lncRNAs}$ and clinicopathological features with significant association were shown in Table 5. Univariate logistic analysis showed that H19, MEG3, and MALAT1 expression were related with HBV infection (all $P<0.10$ ). Multivariate model demonstrated that H19 and MALAT1 were overexpressed in HBV-infected patients $(\mathrm{OR}=1.14,95 \%$ $\mathrm{CI}=1.008-1.29$, and $P=0.037$ and $\mathrm{OR}=26.951,95 \%$ $\mathrm{CI}=2.022-359.284$, and $P=0.013$, resp.). Even though MEG3, HULC, and UCA1 were all associated with vascular invasion in univariate regression analysis (all $P<0.10$ ), only HULC had a significant association with vascular invasion by multivariate model $(\mathrm{OR}=0.648,95 \% \mathrm{CI}=0.523-0.803$, and $P<0.001$ ). Moreover, lncRNAs including H19, MEG3, HULC, HOXA13, and KCNQ1OT1 were all factors associated with AFP level over $200 \mathrm{ng} / \mathrm{mL}$ (all $P<0.10$ ), and when all these lncRNAs were evaluated by a multivariate model using forward selection, H19 and MEG3 were both considered to 


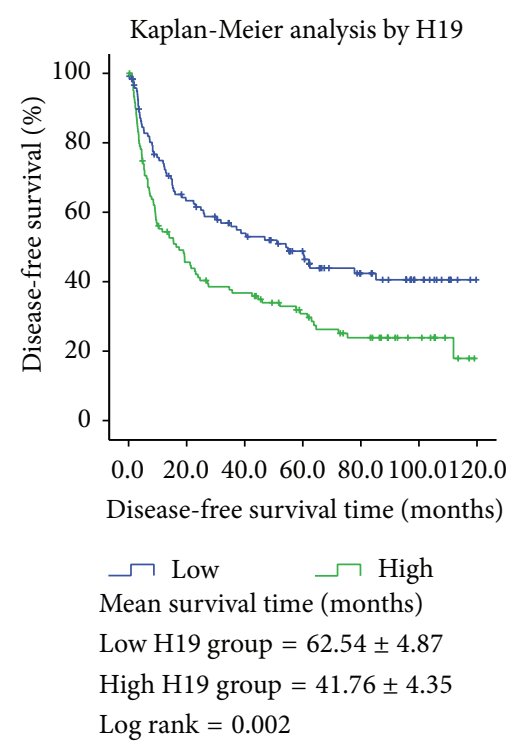

(A)

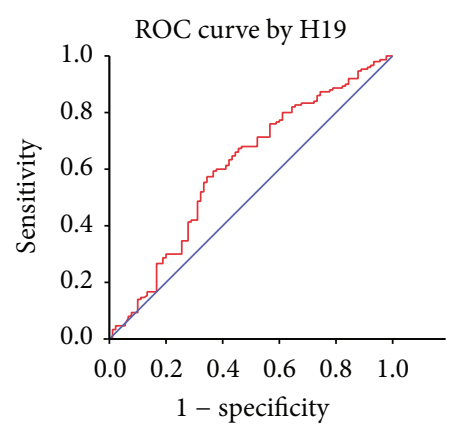

Area under ROC $=0.608$ $P=0.005$

(a)

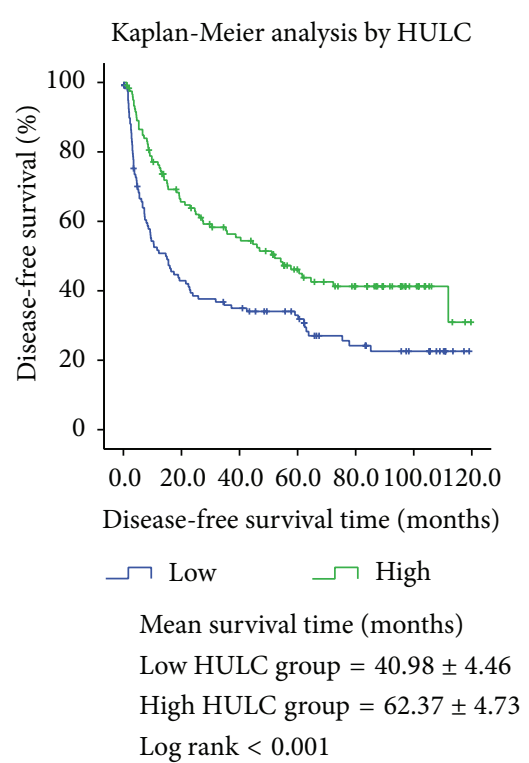

(B)

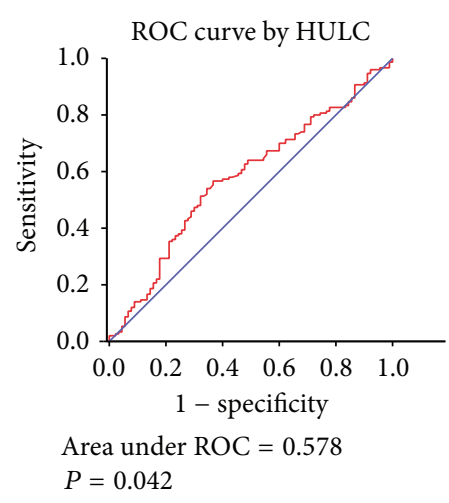

(b)

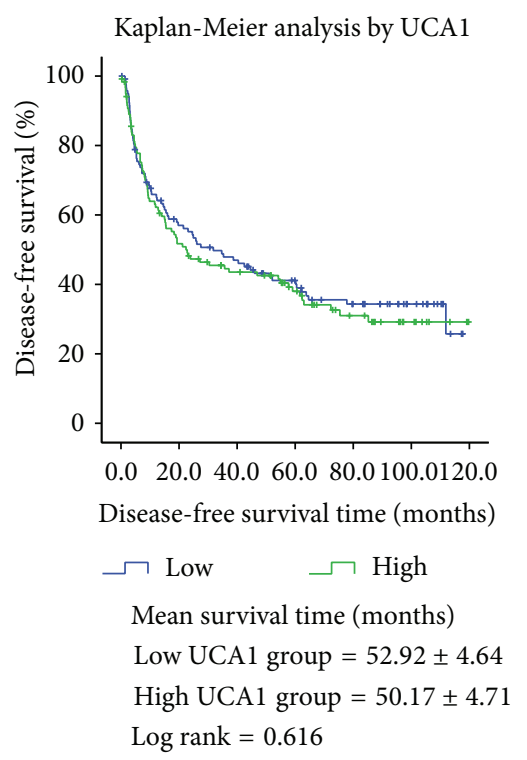

(C)

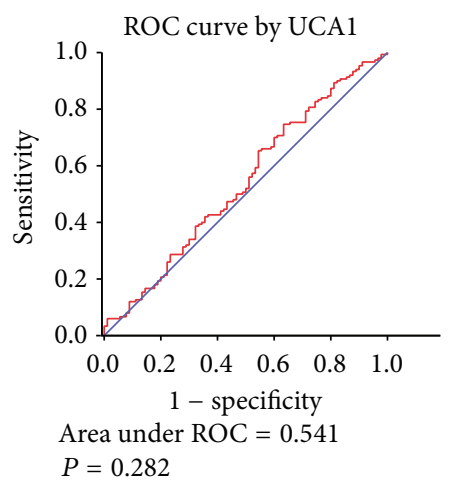

(c)

FIGURE 2: Kaplan-Meier analysis of HCC disease-free survival by H19 (A), HULC (B), and UCA1 (C); ROC curves of H19 (a), HULC (b), and UCA1 (c) for predicting HCC disease-free survival.

be risk factors for high AFP level (OR $=1.45,95 \% \mathrm{CI}=1.277-$ 1.646 , and $P<0.001$ and $\mathrm{OR}=1.613,95 \% \mathrm{CI}=1.1-2.365$, and $P=0.014$, resp.).

\section{Discussion}

Up to date, over 3,000 lncRNAs have been identified and only a small number of functional lncRNAs have been well characterized. Previous studies showed that lncRNAs are likely to be involved in many diverse biological processes, including cell proliferation, differentiation, cell cycle, apoptosis and invasion, marker of cell fate, and parental imprinting, indicating that they may play a major role in the regulation of eukaryotic genome [17-19]. Moreover, multiple lines of evidence link dysregulation of these lncRNAs to diverse human diseases, especially cancers [8-10]. Four known molecular functions of LncRNAs, including signal, decoy, guide, and scaffold, were summarized recently [14]: Firstly, lncRNAs can act as markers of functionally significant biological events by regulating transcriptional activity or pathway. Secondly, lncRNAs can bind and titrate away proteins or RNAs to indirectly exert biological functions in multiple kingdoms of life. Thirdly, the guide function of lncRNAs is that RNA binds specific protein(s) and then directs the localization of the resultant complex to specific targets. Fourthly, lncRNAs can serve as adaptors to bind relevant molecular components to regulate gene expression.

HULC (highly upregulated in liver cancer), $1.6 \mathrm{k}$ nucleotide long, containing two exons but not translated, has been identified highly upregulated in HCC and colorectal cancer that metastasized to livers $[20,21]$. The expression level of HULC is positively correlated with those of $\mathrm{HBx}$ in clinical HCC tissues. Additionally, $\mathrm{HBx}$ could upregulate HULC expression in human immortalized normal liver L-O2 cells and hepatoma HepG2 cells and upregulation of HULC by HBx could promote proliferation of hepatoma cells through suppressing p18 [22]. Based on the previous reports, HULC plays an important role in liver carcinogenesis 
TABLE 5: Univariate and multivariate regression analysis of relationship between lncRNAs and HCC clinicopathological features.

\begin{tabular}{|c|c|c|c|c|}
\hline LncRNAs & $\begin{array}{l}\text { Univariate analysis, } \\
\text { OR }(95 \% \mathrm{CI})\end{array}$ & $P$ value & $\begin{array}{c}\text { Multivariate analysis, } \\
\text { OR }(95 \% \mathrm{CI})\end{array}$ & $P$ value \\
\hline \multicolumn{5}{|c|}{ HBV infection } \\
\hline $\mathrm{H} 19$, per increase of 1 unit & $1.143(1.013-1.29)$ & 0.03 & $1.14(1.008-1.29)$ & 0.037 \\
\hline $\begin{array}{l}\text { MEG3, per increase of } 1 \\
\text { unit }\end{array}$ & $1.503(0.949-2.383)$ & 0.083 & & \\
\hline $\begin{array}{l}\text { MALAT1, per increase of } 1 \\
\text { unit }\end{array}$ & $\begin{array}{c}28.164 \\
(2.179-364.071) \\
\end{array}$ & 0.011 & $\begin{array}{c}26.951 \\
(2.022-359.284) \\
\end{array}$ & 0.013 \\
\hline \multicolumn{5}{|c|}{ Vascular invasion } \\
\hline $\begin{array}{l}\text { MEG3, per increase of } 1 \\
\text { unit }\end{array}$ & $1.425(1.034-1.963)$ & 0.031 & & \\
\hline $\begin{array}{l}\text { HULC, per increase of } 1 \\
\text { unit }\end{array}$ & $0.64(0.522-0.786)$ & $<0.001$ & $0.648(0.523-0.803)$ & $<0.001$ \\
\hline UCA1, per increase of 1 unit & $2.952(0.822-10.601)$ & 0.097 & & \\
\hline \multicolumn{5}{|c|}{ AFP over $200 \mathrm{ng} / \mathrm{mL}$} \\
\hline $\mathrm{H} 19$, per increase of 1 unit & $1.526(1.352-1.723)$ & $<0.001$ & $1.45(1.277-1.646)$ & $<0.001$ \\
\hline $\begin{array}{l}\text { MEG3, per increase of } 1 \\
\text { unit }\end{array}$ & $2.304(1.584-3.351)$ & $<0.001$ & $1.613(1.1-2.365)$ & 0.014 \\
\hline $\begin{array}{l}\text { HULC, per increase of } 1 \\
\text { unit }\end{array}$ & $0.855(0.726-1.007)$ & 0.06 & & \\
\hline $\begin{array}{l}\text { HOXA13, per increase of } 1 \\
\text { unit }\end{array}$ & $0.547(0.288-1.039)$ & 0.065 & & \\
\hline $\begin{array}{l}\text { KCNQ1OT1, per increase of } \\
1 \text { unit }\end{array}$ & $2.557(0.992-6.592)$ & 0.052 & & \\
\hline
\end{tabular}

HBV: hepatitis B virus; AFP: $\alpha$-fetoprotein; OR: odd ratios; CI: confidence intervals.

and acts as an oncogenic ncRNA, but the role of HULC in predicting outcomes in HCC patients after curative therapy was largely unknown. In this study, we found that HULC was elevated in HCC tumor tissues compared with the corresponding nontumor tissues, even though there was no significance. Interestingly, HULC decreased HCC vascular invasion, which should be a positive factor for HCC prognosis. Furthermore, univariate and multivariate Cox regression analyses showed that upregulation of HULC in tumor tissues contributed to better outcomes both in overall survival and in disease-free survival. An interesting report by Liu et al. [10] elucidated that the variant genotypes of rs7763881 in HULC might contribute to decreased HCC susceptibility in HBV persistent carriers. Thus, full molecular mechanism of HULC in the natural history of HBV infection to HCC development should be investigated further.

The H19 gene encodes a $2.3 \mathrm{~kb}$ lncRNA that is exclusively expressed from the maternal allele, and it plays an important role in genomic imprinting during growth and development $[14,23]$. Compared with healthy tissues, H19 is overexpressed in breast adenocarcinoma and is significantly associated with tumor values [24]. Recently, H19 was found to play an important role in HCC progression. In our analysis, H19 was overexpressed in HCC nontumor tissues. Moreover, correlated to chronic HBV infection and AFP evaluation, H19 overexpression was significantly associated with poor disease-free survival. That is, H19 might be a potential biomarker for HCC recurrence prediction. Previous study revealed that $\mathrm{H} 19$ was upregulated in $\mathrm{HBV}$-associated
HCC [14], which is inconsistent with our finding. Since hypoxia is a key trigger enhancing the expression of the H19 gene [25], the fact that more than half of HCC recurrence patients in this study were treated with transarterial chemoembolization (TACE, 86/240) and/or radiofrequency ablation (RFA, 38/240) might be an explanation for this. In vitro, a publication strongly suggested that $\mathrm{H} 19$ could act as a tumor suppressor [26], while other authors assumed that H19 acts as an oncogenic marker in humans $[25,27]$. Although its role in tumorigenesis is debated, the prevailing view is that $\mathrm{H} 19$ behaves like an oncogene [28]. On the other side, AFP is the most widely tested biomarker in HCC. It is known that persistently elevated AFP levels can be used to help define atrisk populations and predict HCC recurrence [29], and H19 mRNA was coregulated with AFP in liver [30]. Considering previous reports and our results, we assumed that $\mathrm{H} 19$ should be a predictive marker for HCC recurrence.

UCA1, short for urothelial cancer associated 1 , is believed to regulate the expression of several genes involved in tumorigenesis, embryonic development, or both [31]. It has been presented that UCA1 functions in regulation of embryonic development and in bladder cancer invasion and progression $[32,33]$, as well as breast tumor [34] and colorectal cancer [35]. Also, overexpression of UCA1 lncRNA could promote metastatic but not proliferation ability of tongue squamous cell carcinoma cells [36]. Our result showed that UCA1 is negatively associated with HCC disease-free survival. Considering no difference in UAC1 expression was found between tumor tissues and nontumor tissues in HCC patients, the 
predictive role of UAC1 in HCC disease-free survival should be evaluated further.

In most of human cancer cell lines, overexpression of MEG3 (maternally expressed gene 3) results in growth suppression, accumulation of $\mathrm{p} 53$ protein, and activation of p53 downstream targets [14]. In HCC tissues and cell lines, MEG3 expression is markedly reduced. It has been well revealed that the loss of MEG3 gene expression is associated with hypermethylation of the promoter region in HCC. Importantly, enforced expression of MEG3 in HCC cells significantly decreases both anchorage-dependent and anchorage-independent cell growth and induces cell apoptosis [37, 38]. However, our research showed that MEG3 elevated in HCC tumor tissues and correlated with AFP elevation in human. As a tumor suppressor gene, MEG3 is associated with tumorigenesis. An overall hypermethylation in specific MEG3 regions might result in permanent gene transcriptional silencing, consequent loss of its antiproliferative function contributing to oncogenesis [39].

MALAT1 (metastasis-associated lung adenocarcinoma transcript 1) was discovered as a prognostic marker for lung cancer metastasis but also has been linked to several other human tumor entities [40]. In our study, MALAT1 is related with chronic HBV infection, but not associated with HCC outcomes. Previously, MALAT1 was reportedly upregulated in HCC cell lines and clinical tissue samples. In addition, inhibition of MALAT1 in HepG2 cells could effectively reduce cell viability, motility, and invasiveness, while increasing the sensitivity to apoptosis $[8,41]$. Therefore, MALAT1 may play an important role in tumor progression and could be a novel biomarker for predicting tumor prognosis [14].

Additionally, HOXA13 and KCNQ1OT1 were upregulated in HCC tumor tissues. A report by Quagliata et al. [42] revealed $\mathrm{HOX}$ genes deregulation to be involved in hepatocarcinogenesis, and HOXA13 are associated with HCC patients' clinical progression and predict disease outcome. Similarly, KCNQ1OT1 has been shown to be involved in multiple cancers. A short tandem repeat polymorphism within KCNQ1OT1 contributes to hepatocarcinogenesis, indicating that common genetic changes in KCNQ1OT1 may influence HCC risk [43]. Further functional studies are needed to validate these hypotheses and understand the roles of lncRNAs in HCC progress and prognosis.

In conclusion, contributing to decreased susceptibility to vascular invasion, upregulation of HULC in tumor tissues was positively associated with HCC survival. In contrast, H19, MEG3, and UCA1 might be risk factors for HCC aggressiveness and poor outcomes. Even though this study was based on data from a national data bank and no direct first-hand data were available and a lot of key questions about lncRNAs remain unsolved, exploration on lncRNAs field is shedding new light on our understanding of HCC. In patients with $\mathrm{HBV}$-associated HCC, the expression of lncRNAs in HBV-HCC tissues was changed significantly compared with normal liver tissues, and lncRNAs played a pivotal role in the pathogenesis of HBV-HCC probably by mainly regulating the carcinoma-related signaling pathway and MAPK signaling pathway [44]. Unfortunately, lncRNA expression in healthy tissues was not available in this analysis. Further research focused on comparison between cancerous and normal samples and the potential action mode of lncRNAs must be conducted. In recent years, there is an exponential growth of studies on the biological functions of lncRNAs in human cancers, including HCC [14]. In the future, integration of lncRNA biology into HCC biology may further deepen our understanding of the mechanisms of HCC and provide novel applications for efficient, rapid, and specific diagnosis and effective treatments.

\section{Conflict of Interests}

The authors have declared that no competing interests exist.

\section{Authors' Contribution}

Zongguo Yang and Yunfei Lu contributed equally to this paper.

\section{References}

[1] J. Ferlay, H.-R. Shin, F. Bray, D. Forman, C. Mathers, and D. M. Parkin, "Estimates of worldwide burden of cancer in 2008: GLOBOCAN 2008," International Journal of Cancer, vol. 127, no. 12, pp. 2893-2917, 2010.

[2] S. Whittaker, R. Marais, and A. X. Zhu, "The role of signaling pathways in the development and treatment of hepatocellular carcinoma," Oncogene, vol. 29, no. 36, pp. 4989-5005, 2010.

[3] P. A. Farazi and R. A. DePinho, "Hepatocellular carcinoma pathogenesis: from genes to environment," Nature Reviews Cancer, vol. 6, no. 9, pp. 674-687, 2006.

[4] J. E. Wilusz, H. Sunwoo, and D. L. Spector, "Long noncoding RNAs: functional surprises from the RNA world," Genes and Development, vol. 23, no. 13, pp. 1494-1504, 2009.

[5] J. Zhao, B. K. Sun, J. A. Erwin, J.-J. Song, and J. T. Lee, "Polycomb proteins targeted by a short repeat RNA to the mouse X chromosome," Science, vol. 322, no. 5902, pp. 750-756, 2008.

[6] X. Wang, S. Arai, X. Song et al., "Induced ncRNAs allosterically modify RNA-binding proteins in cis to inhibit transcription," Nature, vol. 454, no. 7200, pp. 126-130, 2008.

[7] M. Huarte and J. L. Rinn, "Large non-coding RNAs: missing links in cancer?" Human Molecular Genetics, vol. 19, no. 2, pp. R152-R161, 2010.

[8] R. Lin, S. Maeda, C. Liu, M. Karin, and T. S. Edgington, "A large noncoding RNA is a marker for murine hepatocellular carcinomas and a spectrum of human carcinomas," Oncogene, vol. 26, no. 6, pp. 851-858, 2007.

[9] C. Ma, K. Nong, H. Zhu et al., "H19 promotes pancreatic cancer metastasis by derepressing let-7's suppression on its target HMGA2-mediated EMT,' Tumor Biology, vol. 35, no. 9, pp. 9163-9169, 2014.

[10] Y. Liu, S. Pan, L. Liu et al., "A genetic variant in long non-coding RNA HULC contributes to risk of HBV-related hepatocellular carcinoma in a Chinese population," PLoS ONE, vol. 7, no. 4, Article ID e35145, 2012.

[11] E. A. Gibb, C. J. Brown, and W. L. Lam, "The functional role of long non-coding RNA in human carcinomas," Molecular Cancer, vol. 10, article 38, 2011. 
[12] K. C. Wang and H. Y. Chang, "Molecular mechanisms of long noncoding RNAs," Molecular Cell, vol. 43, no. 6, pp. 904-914, 2011.

[13] J. R. Prensner and A. M. Chinnaiyan, "The emergence of lncRNAs in cancer biology," Cancer Discovery, vol. 1, no. 5, pp. 391-407, 2011.

[14] Y. He, X.-M. Meng, C. Huang et al., "Long noncoding RNAs: novel insights into hepatocelluar carcinoma," Cancer Letters, vol. 344, no. 1, pp. 20-27, 2014.

[15] Z. Zhu, X. Gao, Y. He et al., "An insertion/deletion polymorphism within RERT-IncRNA modulates hepatocellular carcinoma risk," Cancer Research, vol. 72, no. 23, pp. 6163-6172, 2012.

[16] H.-Y. Lim, I. Sohn, S. Deng et al., "Prediction of diseasefree survival in hepatocellular carcinoma by gene expression profiling," Annals of Surgical Oncology, vol. 20, no. 12, pp. 37473753, 2013.

[17] T. R. Mercer, M. E. Dinger, and J. S. Mattick, "Long non-coding RNAs: insights into functions," Nature Reviews Genetics, vol. 10, no. 3, pp. 155-159, 2009.

[18] J. Wang, X. Liu, H. Wu et al., "CREB up-regulates long non-coding RNA, HULC expression through interaction with microRNA-372 in liver cancer," Nucleic Acids Research, vol. 38, no. 16, pp. 5366-5383, 2010.

[19] R. A. Gupta, N. Shah, K. C. Wang et al., "Long non-coding RNA HOTAIR reprograms chromatin state to promote cancer metastasis," Nature, vol. 464, no. 7291, pp. 1071-1076, 2010.

[20] K. Panzitt, M. M. O. Tschernatsch, C. Guelly et al., "Characterization of HULC, a novel gene with striking up-regulation in hepatocellular carcinoma, as noncoding RNA," Gastroenterology, vol. 132, no. 1, pp. 330-342, 2007.

[21] I. J. Matouk, I. Abbasi, A. Hochberg, E. Galun, H. Dweik, and M. Akkawi, "Highly upregulated in liver cancer noncoding RNA is overexpressed in hepatic colorectal metastasis," European Journal of Gastroenterology and Hepatology, vol. 21, no. 6, pp. 688-692, 2009.

[22] Y. Du, G. Kong, X. You et al., "Elevation of highly upregulated in liver cancer (HULC) by hepatitis B virus X protein promotes hepatoma cell proliferation via down-regulating p18," The Journal of Biological Chemistry, vol. 287, no. 31, pp. 2630226311, 2012.

[23] M. P. Hitchins and G. E. Moore, "Genomic imprinting in fetal growth and development," Expert Reviews in Molecular Medicine, vol. 4, no. 11, pp. 1-19, 2002.

[24] E. Adriaenssens, L. Dumont, S. Lottin et al., "H19 overexpression in breast adenocarcinoma stromal cells is associated with tumor values and steroid receptor status but independent of p53 and Ki-67 expression," American Journal of Pathology, vol. 153, no. 5, pp. 1597-1607, 1998.

[25] I. J. Matouk, N. DeGroot, S. Mezan et al., "The H19 non-coding RNA is essential for human tumor growth," PLoS ONE, vol. 2, no. 9, article e845, 2007.

[26] Y. Hao, T. Crenshaw, T. Moulton, E. Newcomb, and B. Tycko, "Tumour-suppressor activity of H19 RNA," Nature, vol. 365, no. 6448, pp. 764-765, 1993.

[27] I. Ariel, M. Sughayer, Y. Fellig et al., "The imprinted H19 gene is a marker of early recurrence in human bladder carcinoma," Molecular Pathology, vol. 53, no. 6, pp. 320-323, 2000.

[28] I. J. Matouk, E. Raveh, R. Abu-lail et al., "Oncofetal H19 RNA promotes tumor metastasis," Biochimica et Biophysica Acta, vol. 1843, no. 7, pp. 1414-1426, 2014.
[29] European Association for the Study of the Liver and European Organisation for Research and Treatment of Cancer, "EASLEORTC clinical practice guidelines: management of hepatocellular carcinoma," Journal of Hepatology, vol. 56, no. 4, pp. 908943, 2012.

[30] V. Pachnis, A. Belayew, and S. M. Tilghman, "Locus unlinked to $\alpha$-fetoprotein under the control of the murine raf and Rif genes," Proceedings of the National Academy of Sciences of the United States of America, vol. 81, no. 17, pp. 5523-5527, 1984.

[31] K. Kaneko, Y. Ito, Y. Ono et al., "Gene expression profiling reveals upregulated UCA1 and BMF in gallbladder epithelia of children with pancreaticobiliary maljunction," Journal of Pediatric Gastroenterology and Nutrition, vol. 52, no. 6, pp. 744750, 2011.

[32] F. Wang, X. Li, X. Xie, L. Zhao, and W. Chen, "UCA1, a nonprotein-coding RNA up-regulated in bladder carcinoma and embryo, influencing cell growth and promoting invasion," FEBS Letters, vol. 582, no. 13, pp. 1919-1927, 2008.

[33] C. Yang, X. Li, Y. Wang, L. Zhao, and W. Chen, "Long noncoding RNA UCA1 regulated cell cycle distribution via CREB through PI3-K dependent pathway in bladder carcinoma cells," Gene, vol. 496, no. 1, pp. 8-16, 2012.

[34] J. Huang, N. Zhou, K. Watabe et al., "Long non-coding RNA UCA1 promotes breast tumor growth by suppression of $\mathrm{p} 27$ (Kip1)," Cell Death and Disease, vol. 5, no. 1, Article ID e1008, 2014.

[35] Y. Han, Y.-N. Yang, H.-H. Yuan et al., "UCA1, a long noncoding RNA up-regulated in colorectal cancer influences cell proliferation, apoptosis and cell cycle distribution," Pathology, vol. 46, no. 5, pp. 396-401, 2014.

[36] Z. Fang, L. Wu, L. Wang, Y. Yang, Y. Meng, and H. Yang, "Increased expression of the long non-coding RNA UCA1 in tongue squamous cell carcinomas: a possible correlation with cancer metastasis," Oral Surgery, Oral Medicine, Oral Pathology and Oral Radiology, vol. 117, no. 1, pp. 89-95, 2014.

[37] C. Braconi, T. Kogure, N. Valeri et al., "MicroRNA-29 can regulate expression of the long non-coding RNA gene MEG3 in hepatocellular cancer," Oncogene, vol. 30, no. 47, pp. 4750-4756, 2011.

[38] S. L. Anwar, T. Krech, B. Hasemeier et al., "Loss of imprinting and allelic switching at the DLK1-MEG3 locus in human hepatocellular carcinoma," PLoS ONE, vol. 7, no. 11, Article ID e49462, 2012.

[39] L. Benetatos, G. Vartholomatos, and E. Hatzimichael, "MEG3 imprinted gene contribution in tumorigenesis," International Journal of Cancer, vol. 129, no. 4, pp. 773-779, 2011.

[40] T. Gutschner, M. Hämmerle, and S. Diederichs, "MALAT1-a paradigm for long noncoding RNA function in cancer," Journal of Molecular Medicine, vol. 91, no. 7, pp. 791-801, 2013.

[41] M.-C. Lai, Z. Yang, L. Zhou et al., "Long non-coding RNA MALAT-1 overexpression predicts tumor recurrence of hepatocellular carcinoma after liver transplantation," Medical Oncology, vol. 29, no. 3, pp. 1810-1816, 2012.

[42] L. Quagliata, M. S. Matter, S. Piscuoglio et al., "Long noncoding RNA HOTTIP/HOXA13 expression is associated with disease progression and predicts outcome in hepatocellular carcinoma patients," Hepatology, vol. 59, no. 3, pp. 911-923, 2014.

[43] J. Wan, M. Huang, H. Zhao et al., "A novel tetranucleotide repeat polymorphism within KCNQ1OT1 confers risk for hepatocellular carcinoma," DNA and Cell Biology, vol. 32, no. 11, pp. 628634, 2013. 
[44] Y.-F. Pan, T. Qin, L. Feng, and Z.-J. Yu, "Expression profile of altered long non-coding RNAs in patients with HBV-associated hepatocellular carcinoma," Journal of Huazhong University of Science and Technology-Medical Science, vol. 33, no. 1, pp. 96101, 2013. 


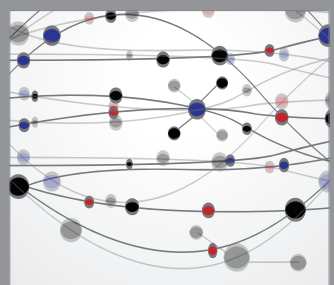

The Scientific World Journal
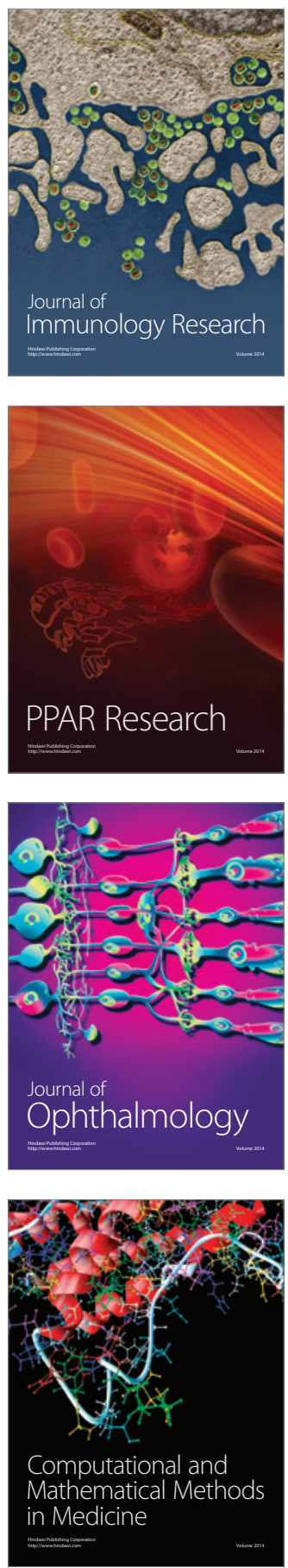

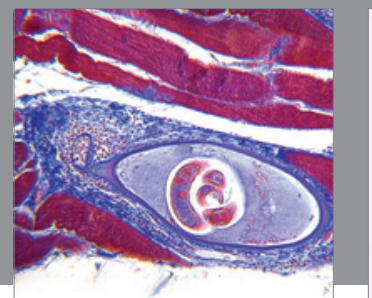

Gastroenterology

Research and Practice
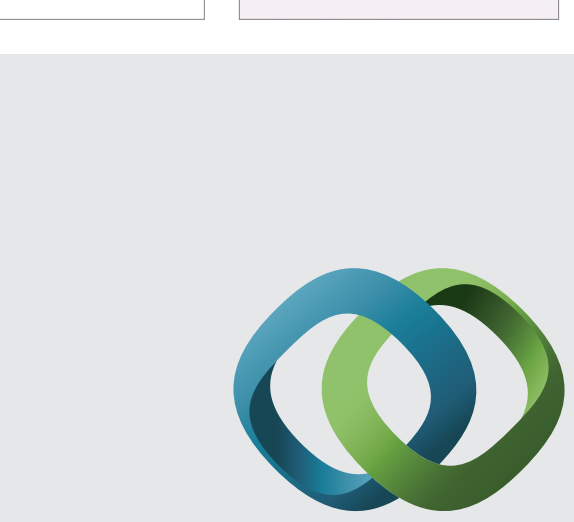

\section{Hindawi}

Submit your manuscripts at

http://www.hindawi.com
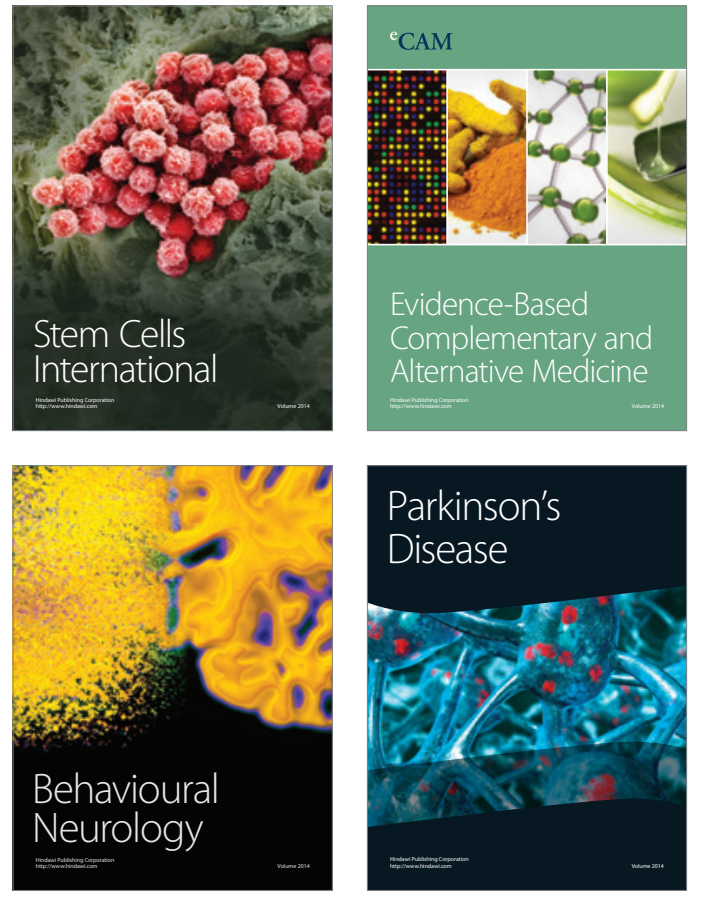
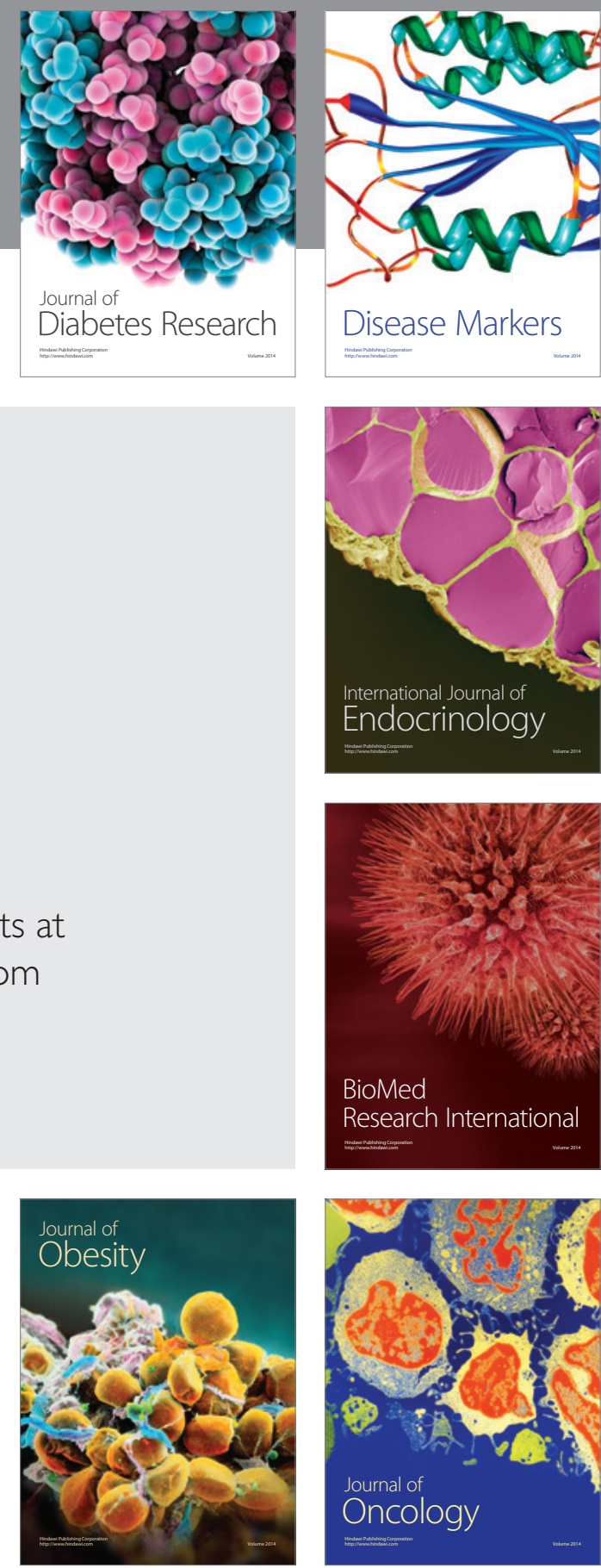

Disease Markers
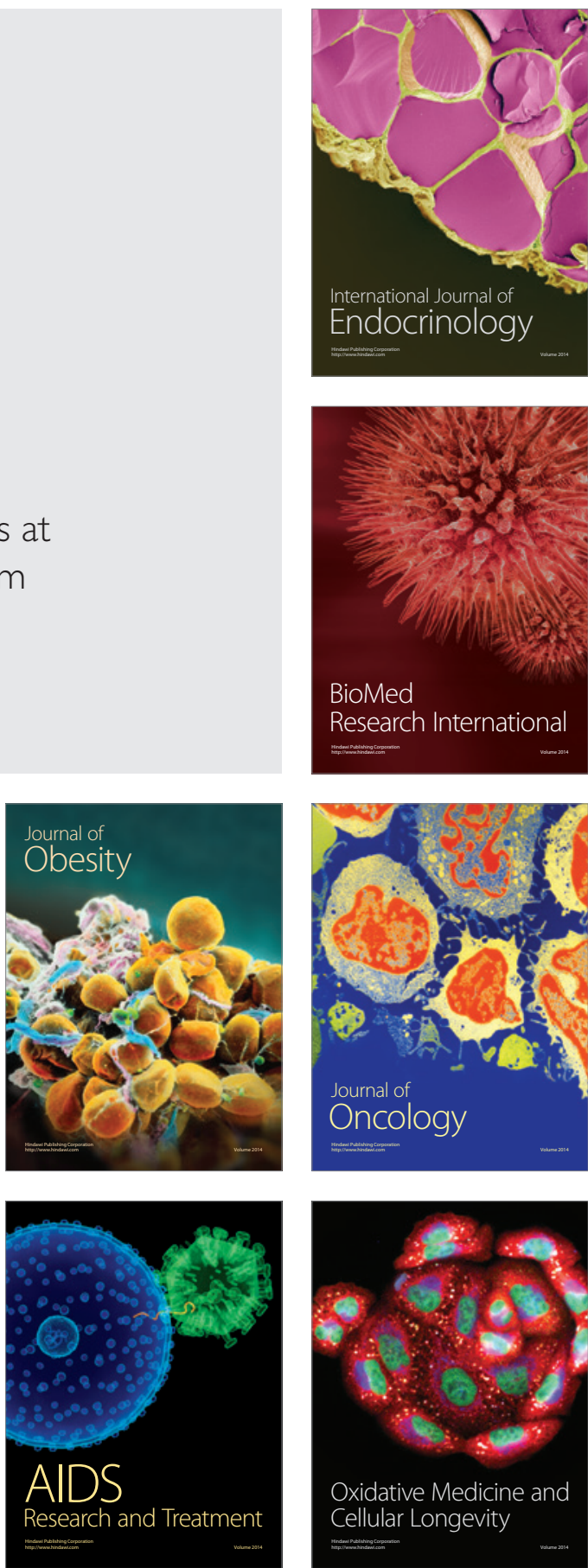